\title{
O QUE TEM NO RIO PROFESSOR? ATIVIDADE INVESTIGATIVA SOBRE MACROINVERTEBRADOS NO ENSINO MÉDIO
}

WHAT'S IN THE RIVER, TEACHER? INVESTIGATIVE ACTIVITY ON MACROINVERTEBRATES IN HIGH SCHOOLS

¿QUÉ HAY EN EL RÍO, PROFESOR? ACTIVIDAD DE INVESTIGACIÓN SOBRE MACROINVERTEBRADOS EN LA ESCUELA SECUNDARIA

\section{Virgínia Teodoro \\ (iD) 9}

Mestre Ensino de Biologia

Professora de Educação Básica

virthe@hotmail.com

\section{João Paulo Cunha de \\ Menezes \\ (iD) 9}

Doutor em Ciências

Professor Adjunto, Ensino de

Ciências e Biologia

Docente PROFBIO

jpaulo_bio@hotmail.com

\begin{abstract}
Resumo
Esse trabalho apresenta um estudo qualitativo desenvolvido a partir da implementação de uma atividade investigativa explorando a fauna de macroinvertebrados em fontes de água com diferentes níveis de poluição. Os dados aqui apresentados e discutidos foram coletados por meio de gravações em áudio das atividades e diário de campo da pesquisadora. Sua análise objetivou investigar como o uso de uma atividade investigativa pode contribuir para a melhoria do ensino de Ciências. Os resultados apontaram elementos que indicam a contribuição da atividade no engajamento e interesse dos estudantes nas atividades desenvolvidas, do mesmo modo que auxiliou na compreensão dos conteúdos abordados.

Palavras-chave: Investigação. Ensino de Ciências. Qualidade da água.
\end{abstract}

Recebido em: 15 de março de 2021.

Aprovado em: 19 de maio de 2021.

Como citar esse artigo (ABNT):

TEODORO, Virgínia; MENEZES, João Paulo Cunha de. O que tem no rio professor? Atividade investigativa sobre macroinvertebrados no ensino médio. Revista Prática Docente, v. 6, n. 2, e035, 2021. http://doi.org/10.23926/RPD.2021.v6.n2.e035.id1048 


\section{Abstract}

This paper presents a qualitative study developed from the implementation of an Investigative Activity exploring the macroinvertebrate fauna in water sources with different levels of pollution. The data presented and discussed here were collected through audio recordings of the activities and the field diary of the researcher. Its analysis aimed to investigate how the use of an Investigative Activity can contribute to the improvement of science teaching. The results pointed out elements that indicate the contribution of the activity in the engagement and interest of students in the developed activities, in the same way that it helped in the understanding of the addressed contents.

Keywords: Research. Science teaching. Water quality.

\section{Resumen}

Este trabajo presenta un estudio cualitativo desarrollado a partir de la realización de una Actividad de Investigación que explora la fauna de macroinvertebrados en fuentes de agua con diferentes niveles de contaminación. Los datos presentados y discutidos aquí se recogieron mediante grabaciones de audio de las actividades y el diario de campo del investigador. Su análisis pretendía investigar cómo el uso de una actividad de investigación puede contribuir a la mejora de la enseñanza de las ciencias. Los resultados señalaron elementos que indican la contribución de la actividad en el compromiso e interés de los estudiantes en las actividades desarrolladas, de la misma manera que ayudaron en la comprensión de los contenidos abordados.

Palabras clave: Investigación. La enseñanza de las ciencias. Calidad del agua. 


\section{INTRODUÇÃOO}

Um dos tópicos mais comentados hoje na Educação Científica tem sido as atividades investigativas. Atividade investigativa é um processo de interação entre professor e estudante em que o professor envolve os alunos na geração de perguntas e na busca de respostas por meio de cuidadosa observação e reflexão (CARVALHO, 2013). Essas atividades podem ser definidas como um processo de descobertas de novas relações causais, com o estudante formulando hipóteses e testando-as, realizando experimentos e/ou observações (PEDASTE et al., 2012). Desta forma, os estudantes geralmente realizam um processo de aprendizagem autodirigido, parcialmente indutivo e parcialmente dedutivo, fazendo experimentos ou observações para investigar as relações de um ou mais conjunto de variáveis (WILHELM; BEISHUIZEN, 2003).

As atividades investigativas proporcionam aos estudantes um "autêntico" processo de descoberta científica. Do ponto de vista pedagógico, este processo por ser dividido em unidades menores, conectadas, orientam os estudantes para características importantes do pensamento científico (PEDASTE et al., 2015). Neste contexto, a literatura apresenta uma diversidade de modelos, divididos em fases e ciclos de investigação a ser trabalhado. Por exemplo, Bybee et al. (2006) propõem um modelo com cinco fases de investigação: engajamento, exploração, explicação, elaboração e avaliação. Em outro modelo, Puche e Holt (2012) propõem um ciclo com 4 fases: pergunta, ação, reflexão e pergunta. Neste ciclo, os alunos ajudam a decidir o que comparar, o que medir e como medir e coletar as informações/dados (PUCHE; HOLT, 2012). No processo de reflexão, os estudantes explicam seus resultados e deliberam sobre o que modificar para uma próxima vez, o que proporciona novos questionamentos.

Para estimular os estudantes nas atividades investigativas Novak e Treagust, (2018) articulam que o professor deve envolver seus estudantes com o fenômeno a ser trabalhado, explorando questões fundamentais sobre o mundo natural por meio de aprendizagem tridimensionais, no qual o estudante tem acesso ao objeto estudado. À medida que os estudantes se envolvem nessas atividades, eles começam a observar a importância das atividades científicas para enfrentar os principais desafios que a sociedade moderna enfrenta. Um desses desafios é a manutenção da qualidade da água. Envolver os estudantes em um projeto relacionado à qualidade da água cria um contexto ideal para trabalhar práticas, desenvolvendo ideias científicas.

Puche e Holt (2012) utilizaram de uma atividade investigativa explorando a fauna de macroinvertebrados relacionados a qualidade da água em sala de aula. Nesta atividade, as 
autoras concluem que o uso de macroinvertebrados pode ser uma excelente ferramenta para despertar os interesses dos estudantes na descoberta científica. Comportamento semelhante foi observado por Novak e Treagust (2018) no qual trabalhou durante quatro semanas a qualidade de água de um riacho por meio de uma atividade investigativa com estudantes do ensino fundamental. Esses autores relatam em teu trabalho que, os alunos ao investigarem um fenômeno complexo, a qualidade da água de um riacho, propiciou a criação de várias interações e explicação sobre o fenômeno envolvido.

Considerando a relevância da utilização de representação no ensino de Ciências e a realização de atividades investigativas, as quais, segundo pesquisas (WILHELM; BEISHUIZEN, 2003; PEDASTE et al., 2012; PUCHE; HOLT, 2012; CARVALHO, 2013; PEDASTE et al., 2015), proporcionam engajamento e possibilitam aos estudantes melhor reflexão sobre o conteúdo, neste estudo, objetivou-se investigar quais significados os estudantes do ensino médio constroem sobre as diferenças de organismo quando desenvolvem uma atividade de investigação semi-guiada. Assim, desenvolvemos uma atividade semi-guiada baseada em perguntas em sala de aula e no campo para comparar a abundância da fauna de macroinvertebrados em riacho com diferentes níveis de poluição.

\section{MATERIAIS E MÉTODOS}

A pesquisa apresentada nesse trabalho tem característica qualitativa, tal escolha ocorreu devido ao propósito de investigar as contribuições de uma atividade investigativa para o ensino de Ciências. Os dados coletados são, em sua maioria, descritivos, sendo o interesse tanto pelo processo quanto pelos resultados obtidos. Para um bom desenvolvimento, a pesquisa tem como ideia essencial, a imersão na esfera da subjetividade e do simbolismo, presente no contexto social e cultural dos estudantes. Para Bodgan e Biklen (1994), a pesquisa qualitativa possibilita que o pesquisador se insira na sala de aula e participe da aplicação das atividades não apenas como observador, mas participante do processo que está investigando. Assim, “do ponto de vista metodológico, a melhor maneira para se captar a realidade é aquela que possibilita ao pesquisador colocar-se no papel do outro, vendo o mundo pela visão dos pesquisados" (GODOY, 1995, p. 57).

Participaram da atividade 25 estudantes do $1^{\circ}$ ano do ensino médio da Escola Estadual Doutor Virgílio de Melo Franco, em Paracatu-MG/Brasil. As atividades da pesquisa desenvolveram-se em três momentos: i) saída de campo, coleta de amostras dos riachos seguindo por classificação dos habitats. A classificação dos habitats foi utilizada para que o 
estudante compreenda as diferenças de cada ponto, ii) logo após, na escola foi realizado a identificação dos organismos (estudantes e professora) e a proposição de uma situação problema com construção das hipóteses e, no terceiro momento iii) a socialização dos resultados. A atividade desenvolvida foi elaborada, considerando as características da investigação propostas por Bybee (2006).

No primeiro momento houve uma saída de campo guiada para a coleta de macroinvertebrados. Os estudantes foram separados em 4 grupos distintos para realização das coletas e classificação dos pontos. A coleta foi realizada em um dos braços do corrégo Rico (área impactada por atividades urbanas e rurais) e na nascente Alto do Açude (área com vegetação preservada). Em cada ponto de coleta foi realizada 4 (quatro amostragem) por grupo. Após a coleta dos macroinvertebrados os grupos de estudantes foram solicitados a avaliar o ponto de amostragem utilizando um Protocolo de Avaliação Rápida (PAR) para diversidade de habitats (CALLISTO et al., 2002). Este protocolo avalia um conjunto de parâmetros físicos do meio por meio de uma pontuação entre 0 e 4, que é atribuída a cada indicador com base na observação das condições ambientais locais. Ao final da avaliação as notas de todos os parâmetros são totalizadas, gerando os diagnósticos de qualidade ambiental dos segmentos fluviais, que variam entre "impactados", "alterados" e "naturais".

O segundo momento foi realizado após a saída de campo, em sala de aula no qual os grupos de estudantes juntamente com a professora realizaram a identificação das espécies coletadas a nível de família. Os organismos foram preservados em etanol a 70\% e, em seguida, identificados por meio de literatura especializada (MUGNAI; NESSIMIAN; BAPTISTA, 2010). Após a classificação dos organismos, a professora proporcionou um momento de interação. O objetivo dessa primeira intervenção foi verificar quais significados sobre zoologia os estudantes apresentavam. Logo em seguida, a professora apresentou um problema: por que os organismos presentes nos dois pontos de coleta são diferentes? Como podemos comprovar? Por meio deste questionamento os estudantes puderam avaliar e discutir a situação apresentando possíveis hipóteses.

Em um terceiro momento a professora proporcionou outro tempo de interação dialogada em que discutiram os motivos pela diferença de organismos entre os pontos nas condições observadas. Neste ponto todo conteúdo gerado pelos estudantes foi socializado e discutido. Durante o desenvolvimento das atividades buscou-se, a todo o momento, criar um ambiente 
encorajador para os estudantes, de forma a favorecer a troca de ideias, cooperação e ajuda entre colegas.

Como instrumento de avaliação houve a escrita individual por parte da professora e a gravação áudio visual de todas as ações desenvolvidas ao longo das atividades. De forma a adequar o presente trabalho aos preceitos éticos da pesquisa, os estudantes que aceitaram participar da visita de campo e da pesquisa assinaram de livre e espontânea vontade um Termo de Consentimento Livre e esclarecido. Os participantes da pesquisa foram denominados com a sigla Est. (estudante) seguido de um número (1 a 25).

\section{ReSUltados E DisCUSSÕES}

Em um primeiro momento, antes da saída de campo, a professora iniciou as atividades fazendo uma interação dialógica com os estudantes sobre os macroinvertebrados. Nessa primeira intervenção foram discutidos os aspectos gerais deste grupo a fim de promover situações em que os estudantes pudessem evidenciar os conceitos em campo. Essas atitudes e ações, vivenciadas pela professora e estudantes, são descritas por Carvalho (2013), como importante no ensino em que o estudante constrói seu conhecimento, pois gerarão ideias que ao serem refletidas, possibilitarão ampliar conhecimentos prévios, oportunizarão discussões, estabelecerão métodos de trabalho e construção de significados.

Logo após, já em campo, os estudantes aplicaram Protocolo de Avaliação Rápida obtendo a seguinte pontuação: 30 pontos para o Córrego Rico sendo classificado como "ambiente alterado e 60 pontos para a nascente Alto do Açude, classificado como "ambiente natural". O resultado desta avaliação evidenciou para os estudantes que, apesar do Córrego Rico apresentar características físicas aparentemente boas, este manancial apresenta uma qualidade inferior com relação ao Alto do Açude.

"No Alto do Açude estava mais conservado do que o Córrego Rico" Est. 7

"Na nascente do parque ecológico tinha poluição e interferência do homem, porém tinha melhor qualidade do que o Córrego Rico." Est.13

"Na nascente no Alto do Açude têm menos poluição visível do que no Córrego Rico." Est. 14

Esta metodologia prática permite com que os estudantes possam identificar diversos parâmetros físicos na qualidade dos cursos d'água como um todo (para mais informações ver CALLISTO et al., 2002), levando em considerações atividades antrópicas, bem como as alterações decorrentes da mesma em todo o ambiente (CALLISTO et al., 2002). As diferenças encontradas, em combinação com as observações diretas dos estudantes sobre os pontos 
amostrados, permitiram em sala de aula gerar hipóteses úteis para explicar os dados vinculados a qualidade da água e ao uso da terra. O desempenho dos grupos na aplicação do PAR serviu como uma medida independente para a aprendizagem dos estudantes e forneceu evidências para os ganhos de aprendizagem.

Após a saída de campo, já em sala de aula, os estudantes com ajuda da professora identificaram as amostras de água. Ao todo foram coletados e identificados no Alto do Açude 67 indivíduos (representando 12 famílias) e no Córrego Rico 68 indivíduos (representando 8 famílias). Os estudantes descobriram que havia uma variação entre os organismos encontrados dentro de cada local.

Com as amostras identificadas a professora regente colocou uma problemática para que os estudantes pudessem discutir. A pergunta problema foi: Por que os organismos presentes nos dois pontos de coleta são diferentes? Como podemos comprovar? Durante a discussão de acompanhamento desta atividade, os estudantes levantaram hipóteses sobre o que poderia ser responsável pelas diferenças observadas. Algumas das hipóteses levantadas pelos estudantes seguem abaixo apresentadas:

\footnotetext{
"Tem bichinhos tolerantes a poluição e outros não. As larvas vermelhas (referenciando a família Chironomidae) são mais comuns nos dois. Mais alguns tem só tem no Alto do Açude. E os mais comuns são tolerantes a poluição do córrego" Est. 2

"Os que são comuns nos dois locais é porque toleram as variações das condições ambientais, eles conseguem sobreviver em locais que indicam ter poluições e contaminações. Os bichos que foram encontrados só no Alto do Açude é porque precisam de uma condição ambiental melhor." Est. 3

"Bom! No Alto do Açude estava mais limpo e coletamos algumas espécies que só encontramos lá, então estas espécies, seriam sensíveis a poluição do Córrego Rico, porque não foram encontradas nele. E no córrego encontramos poucas espécies diferentes, então elas seriam toleram a poluição deste local." Est. 6
}

O momento de apresentar suas hipóteses permitiu que os estudantes confrontassem suas ideias uns com os outros, libertando-se da passividade de serem meros executores de instruções, o que favoreceu na construção do conhecimento. Essa concepção é defendida por diversos autores (AZEVEDO, 2009; CARVALHO, 2013), no qual afirmam que, durante ação investigativa, a todo o momento, os estudantes podem interagir uns com os outros. Com a elaboração das hipóteses pelos estudantes, foram evidenciados os seguintes significados iniciais estabelecidos na estrutura de conhecimento: i) variação das condições ambientais; ii) presença de indivíduos tolerantes ao nível de poluição; iii) diversidade de espécies.

A partir dos dados apresentados, é possível verificar que os estudantes do $1^{\circ}$ ano do ensino médio conseguem fazer relações coerentes entre o nível de preservação dos mananciais 
com a diversidade de macroinvertebrados. Os significados já existentes na estrutura de conhecimento dos estudantes apresentaram-se satisfatórios para essa fase de escolaridade. Outro ponto que merece destaque é o fato de os estudantes estarem envolvidos durante toda atividade investigativa, diferentemente do que ocorre tradicionalmente nas aulas. Também foi possível notar que os estudantes tiveram boa participação nas interações discursivas e demonstraram ter significados prévios claros e bem estabelecidos na estrutura de conhecimento quanto ao conceito relações entre a presença dos organismos com as condições de preservação local. Admite-se a importância deste momento de discussão para ativar os processos mentais que possibilitam o raciocínio, sendo este um dos objetivos da atividade investigativa (FREITAS-ZOMPERO; LABURU, 2016).

Os resultados sobre a pergunta: Por que os organismos presentes nos dois pontos de coleta são diferentes? Como podemos comprovar? foram:

“...os locais têm algumas espécies iguais e outras diferentes, pois o ambiente é diferente quanto as condições ambientais" Est. 1

"... porque um tinha mais espécies do que os outros e deve ser por causa das condições ambientais" Est.4

“.. no Alto do Açude estava mais limpo e conservado então as espécies só encontradas lá gostam de lugar limpo" Est. 7

O objetivo deste momento foi promover uma atividade investigativa semi-guiada na sala de aula e incentivar os estudantes a pensar de forma crítica, fazer suas próprias suposições e passar pelo ciclo de investigação utilizando dos macroinvertebrados para avaliar a água.

Freire (1987) defende a importância da problematização do conhecimento pelo professor e enfatiza a necessidade de desafiar os estudantes na construção do conhecimento, pois "quanto mais se problematizam os educandos, como seres no mundo, tanto mais se sentirão desafiados, quanto mais obrigados a responder o desafio" (FREIRE, 1987, p. 70).

Por fim, em um terceiro momento, a professora iniciou a atividade relembrado sobre as discussões geradas. Tal ação objetivou retomar o conhecimento construído nos momentos anteriores. Após este momento a professora proporcionou um tempo para socialização e discussão dos resultados relacionando-os com os conceitos envolvidos com a atividade. Os estudantes discutiram entre eles, e em seguida apresentaram as hipóteses elaboradas. Este comportamento corrobora com a postura apresentada por Carvalho et al. (2007, p. 31) para uma atividade investigativa, no qual "ao contar aos outros o que pensam sobre um problema, os estudantes elaboram e refinam seus pensamentos e aprofundam sua compreensão". Acreditamos que, o papel exercido pela professora na condução da discussão com os estudantes 
promoveu o acesso aos conhecimentos e informações importantes permitindo a adequação das hipóteses e conceitos. Tal situação é exemplificada com as modificações das hipóteses produzidas pelos estudantes, percebendo um amadurecimento e independência dos estudantes. Esta postura permitiu uma cooperação entre os estudantes com a professora favorecendo o protagonismo deles no processo de construção dos conhecimentos científicos. Este comportamento corrobora com o que se espera nas escolas.

Azevedo (2009) ressalta que, ao utilizar de atividades investigativas como ponto de partida para a compreensão de conceitos científicos possibilita aos estudantes a participar de um processo de aprendizagem saindo de uma postura passiva. Driver et al. (2000) completa, afirmando que um estudante que sabe construir seus argumentos ao realizar afirmações sobre fenômenos está aprendendo Ciências. Conforme afirmam Sasseron e Carvalho (2011), a concepção de educação não deve ser apenas instrução e aprendizagem dos conteúdos, mas a formação de uma autonomia crítica do sujeito, por isso é importante oferecer atividades que promovam um processo dialógico.

Além disso, essa experiência contribuiu para aumentar a conscientização dos estudantes sobre as ameaças à comunidade de macroinvertebrados e ampliar suas perspectivas sobre como identificar e lidar com problemas ambientais.

\footnotetext{
"Antes eu pensei que os locais tinham condições ambientais normais, mas depois da análise das condições dos locais, vi que não são boas" Est. 1

"Minha visão foi que devemos evitar de jogar lixo, esgoto, conservar e despoluir os locais já poluídos. Deve-se conserva-los para que tenham maior diversidade de plantas e animais, além de favorecer a água para os seres humanos” Est. 4

“Antes eu achava que o Córrego Rico era mais bem preservado, mais na aula eu observei que está em péssimas condições. E a minha vó fala que era um córrego com muita água e bonito" Est. 12
}

A atividade investigativa com uso de macroinvertebrados favoreceu o interesse dos estudantes e possibilitou conectarem evidências observadas nas atividades com o conhecimento científico, permitindo aos mesmos a produção de significados. Ressaltamos que as modalidades utilizadas não incluíram aulas expositivas. Os significados dos estudantes demostrados foram produzidos apenas com a utilização de saídas de campo e discussões em grupo. Quanto a importância de trabalhar temas ambientais, os resultados deste estudo se somam ao crescente corpo de estudo que indica o valor dos projetos de qualidade da água para o ensino de ciências (JAMES et al., 2006; KOOSMANN et al., 2011; ENOS-BERLAGE, 2012; PUCHE; HOLT, 2012). Significativamente, devido ao grande número de córregos, rios, lagos e oceanos 
poluídos, os locais de projetos para esses tipos de experiências educacionais estão amplamente (e infelizmente) disponíveis.

\section{CONSIDERAÇÕES FINAIS}

Conforme observado, com os resultados da atividade investigativa, verificou-se que os estudantes relacionaram a diversidade de espécies de macroinvertebrados com o nível de poluição. A atividade favoreceu um melhor engajamento dos estudantes possibilitando correlacionarem as evidências observadas com o conhecimento científico, permitindo a elaboração de significados. Desta forma, estudar e discutir a qualidade da água usando macroinvertebrados apresentou-se como uma forma rápida e divertida de incentivar o interesse dos estudantes pela descoberta científica. Apresentando-se como uma forma de alterar a lacuna entre a educação formal e a vida real dos estudantes, trazendo novas perspectiva sobre como identificar problemas ambientais. Acreditamos que, a incorporação de aulas baseadas em atividades investigativas pode promover o pensamento crítico dos estudantes, capacitá-los a se tornarem aprendizes independentes e ao longo da vida, ensiná-los a enfrentar problemas, gerar e testar ideias para si mesmos, e questionar os valores cotidianos e sua compreensão do mundo.

\section{Referências}

AZEVEDO, Maria Cristina P. Stella. Ensino por investigação: problematizando as atividades em sala de aula. In: CARVALHO, Anna Maria Pessoa (Org.). Ensino de Ciências: unindo a pesquisa e a prática. São Paulo: Cengage Learning, 2009.

BOGDAN, Robert; BIKLEN, Sari. Investigação qualitativa em Educação: fundamentos, métodos e técnicas. Investigação qualitativa em educação. Portugal: Porto Editora, p. 1580, 1994.

BYBEE, Rodger W. Scientific inquiry and science teaching. In: Scientific inquiry and nature of science. Springer, Dordrecht, 2006. p. 1-14.

CALLISTO, Marco; FERREIRA, W; MORENO, P.; GOULART, M.; PETRUCIO, M. Aplicação de um protocolo de avaliação rápida da diversidade de habitats em atividade de ensino e pesquisa (MG-RJ). Acta Limnologica Brasiliensia, v. 14, n. 1, p. 91-98, 2002.

CARVALHO, Anna Maria Pessoa; VANNUCCHI. Andréia Infatosi; BARROS, Marcelo Alves; GONÇALVES, Maria Elisa Resende; REY, Renato Casal. Ciências no Ensino Fundamental: o conhecimento físico. São Paulo: Scipione, 2007.

CARVALHO, Anna Maria Pessoa. O ensino de ciências e a proposição de sequências de ensino investigativas. Ensino de ciências por investigação: condições para implementação em sala de aula. São Paulo: Cengage Learning, p. 1-20, 2013. 
DRIVER, Rosalind; ASOKO, Hilary; LEACH, John; MORTIMER, Eduardo; Scott, Philip. Construindo conhecimento científico em sala de aula. Química nova na escola, n. 9, p. 3140, 2000.

ENOS-BERLAGE, Jodi. Development of a water-quality lab that enhances learning \& connects students to the land. The American Biology Teacher, v. 74, n. 7, p. 471-478, 2012.

FREIRE, Paulo. Pedagogia do Oprimido. 17ª edição. Rio de Janeiro: Paz e Terra, p. 259268, 1987.

FREITAS ZOMPERO, Andréia; LABURU, Carlos Eduardo. Significados de fotossíntese apropriados por alunos do ensino fundamental a partir de uma atividade investigativa mediada por multimodos de representação. Investigações em Ensino de Ciências, v. 16, n. 2, p. 179$199,2016$.

GODOY, Arilda Schmidt. Introdução à pesquisa qualitativa e suas possibilidades. RAERevista de Administração de Empresas, v. 35, n. 2, p. 57-63, 1995.

JAMES, Rebecca R.; OGDEN, Albert E.; DIVINCENZO, John P. A water quality study in Rutherford County, Tennessee: student group project. Journal of Natural Resources \& Life Sciences Education, v. 35, n. 1, p. 118-126, 2006.

KOOSMANN, Angelena; BRUNS, Alex; ANTHONY, Sharon. The Chemistry of natural Waters. Science Education \& Civic Engagement, p. 48, 2011.

MUGNAI, Riccardo; NESSIMIAN, Jorge Luiz; BAPTISTA, Darcilio Fernandes. Manual de identificação de macroinvertebrados aquáticos do Estado do Rio de Janeiro: para atividades técnicas, de ensino e treinamento em programas de avaliação da qualidade ecológica dos ecossistemas lóticos. Technical Books Editora, 2010.

NOVAK, Ann M.; TREAGUST, David F. Adjusting claims as new evidence emerges: Do students incorporate new evidence into their scientific explanations?. Journal of Research in Science Teaching, v. 55, n. 4, p. 526-549, 2018.

PEDASTE, Margus; MÄEOTS, Mario; LEIJEN, Äli; SARAPUU, Tago. Improving students' inquiry skills through reflection and self-regulation scaffolds. Technology, Instruction, Cognition and Learning, v. 9, n. 1-2, p. 81-95, 2015.

PUCHE, Helena; HOLT, Jame. Using scientific inquiry to teach students about water quality. The american biology Teacher, v. 74, n. 7, p. 503-508, 2012.

SASSERON, Lúcia Helena; CARVALHO, Anna Maria Pessoa de. Construindo argumentação na sala de aula: a presença do ciclo argumentativo, os indicadores de alfabetização científica e o padrão de Toulmin. Ciência \& Educação, v. 17, n. 1, p. 97-114, 2011.

WILHELM, Pascal; BEISHUIZEN, Jos Beishuizen. Content effects in self-directed inductive learning. Learning and Instruction, v. 13, n. 4, p. 381-402, 2003. 